
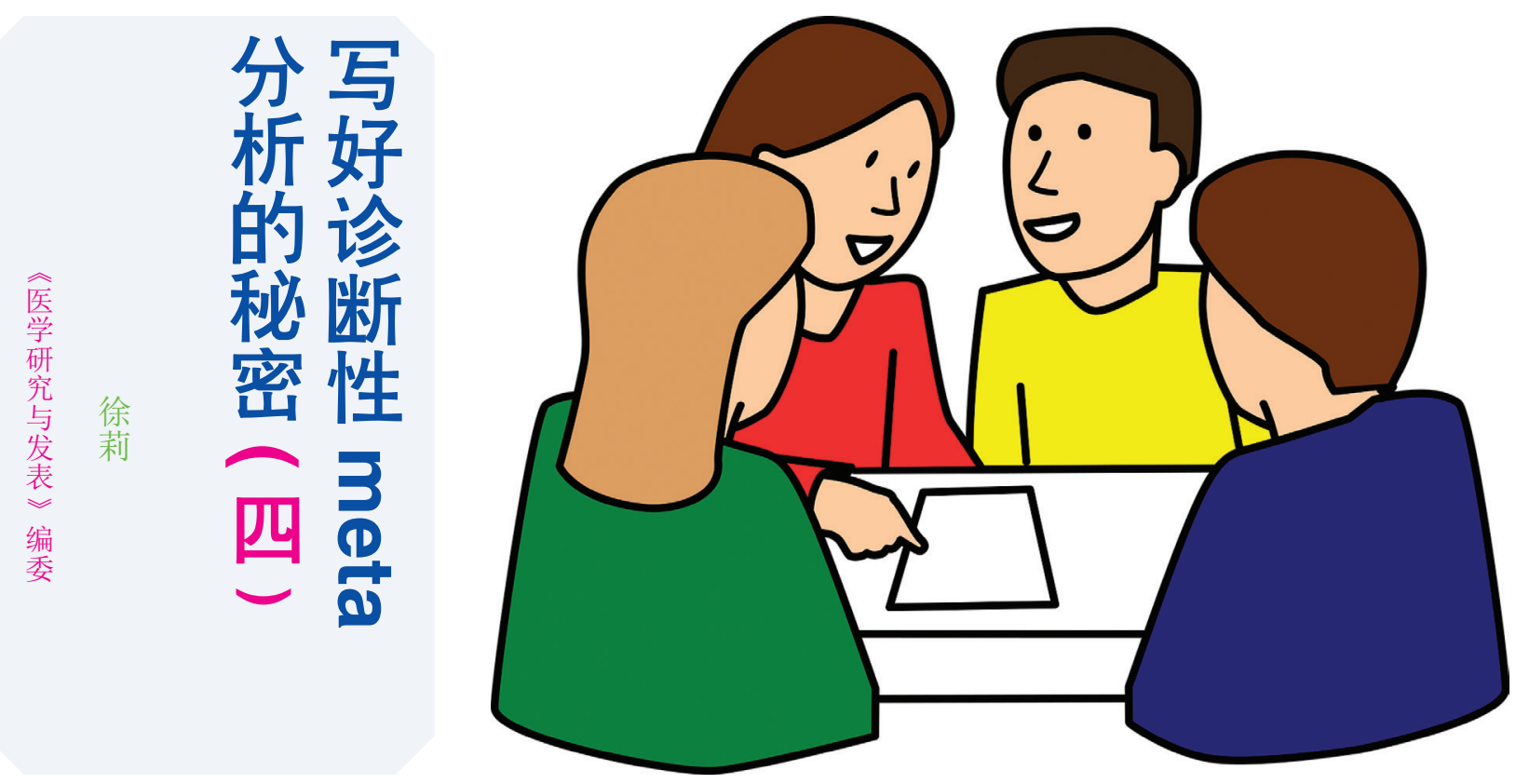

复杂动作怎么做？

方法一分亚组

分亚组的原因, 在何种情况 下需要用到分亚组?

我自己的理解, 就像一幅拍
好的照片需要后处理 -- 如果相片 拍的好, 那么不进行后处理也是 可以的; 如果相片拍的不好, 进 行后处理可以把里面的局部凸显 出来也是可以的, 假如相片拍的 不好, 那么说不定局部还是美的 呢…...
这里的照片拍得好不好—— 可以类比为文章最后出来的结果 好不好。

咱医学生, 最怕看大段大段 的文字了, 为了让大家有一个良好 的阅读体验及直观的理解, 请看 以下图例:

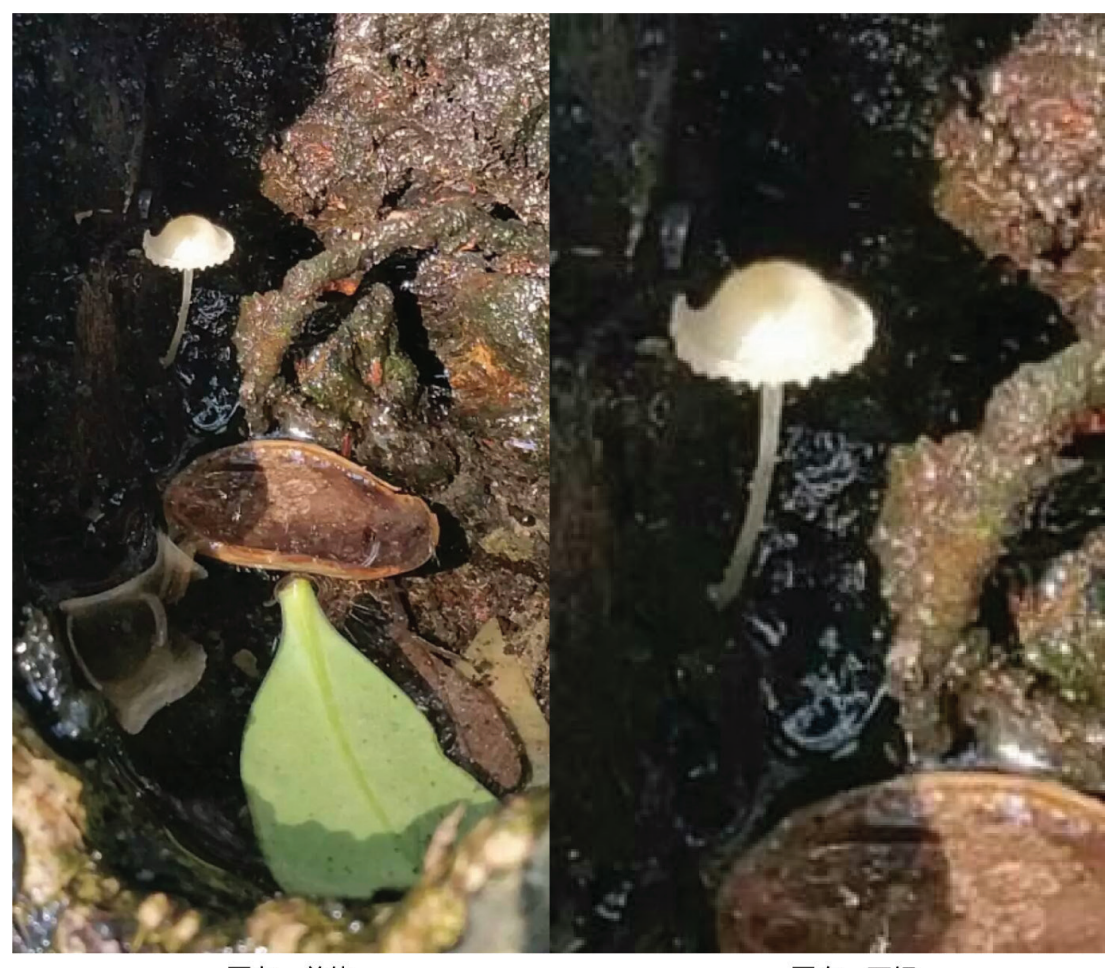


类似的对应我们的诊断性 meta 分析的写作中:

图左一一最基本的诊断性 meta 分析单个技术对肺癌的诊断 价值 (蘑菇花生壳叶子)。

图右一一扩展为单个技术对 非小细胞肺癌 (蘑菇) 的诊断价值 以及单个技术对小细胞肺癌 (磨 菇）的诊断价值 -- 当然此处也不 一定非得是蘑菇, 花生壳和叶子
都可以的, 看看什么更好看就放 什么，文章中也是亦然。

问题: 分亚组需要获得的数 据和具体操作方法。

分亚组需要获得的数据 -- 看 文章 -- 比如要做 DWI 对非小细 胞肺癌的亚组分析 -- 那么在读这 篇文章时候如果文章本来就是以 非小细胞肺癌作为研究对象的自 然可以入选。
具体操作方法 --- 读全文--总之最后要能提取真阳性、假阴 性、假阳性、真阴性的数据才可 以做。

分亚组示例

假如研究 1 及研究 3 为非小 细胞肺癌。

研究 2 研究 4-5 为小细胞肺 癌。

\begin{tabular}{|c|c|c|c|c|c|c|c|}
\hline No. & 恶性病变数目 & 良性病变数目 & 真阳性 & 假阴性 & 假阳性 & 真阴性 & 分组示例 \\
\hline 1 & 36 & 180 & 30 & 4 & 6 & 176 & 非小细胞肺癌 \\
\hline 2 & 26 & 84 & 14 & 11 & 12 & 73 & 小细胞肺癌 \\
\hline 3 & 19 & 24 & 18 & 2 & 1 & 22 & 非小细胞肺癌 \\
\hline$\underline{4}$ & 45 & 34 & 37 & 6 & 8 & 28 & 小细胞肺癌 \\
\hline
\end{tabular}

非小细胞肺癌敏感性

具体操作方法:

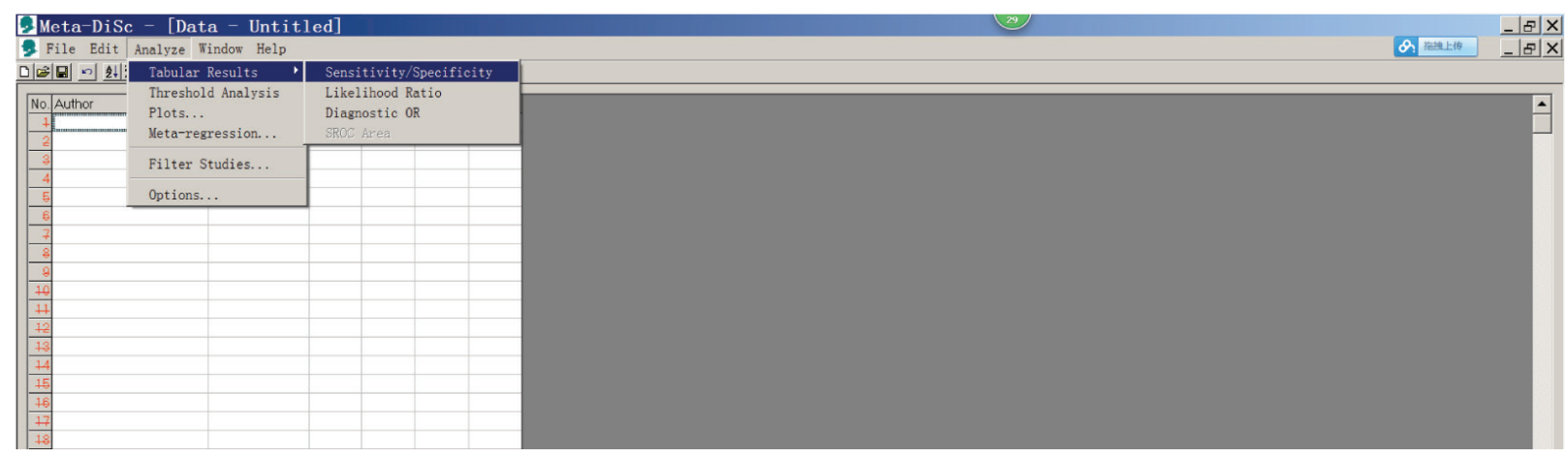

Summary Sensitivity

下划线部分需要记录并在 meta分析结果中报告。

Study | Sen [95\% Conf. Iterval.] TP/(TP+FN) TN/(TN+FP)

$\begin{array}{rrrrr}10.833 & 0.672 & -0.936 & 30 / 36 & 176 / 180 \\ 10.947 & 0.740 & -0.999 & 18 / 19 & 22 / 24\end{array}$

\begin{tabular}{llll} 
Pooled Sen & $0.873 \quad 0.755-0.947$ \\
\hline
\end{tabular}

Heterogeneity chi-squared $=1.65$ (d.f.= 1) $p=0.199$

Inconsistency (I-square) $=39.5 \%$

No. studies $=2$.

Filter OFF

Add $1 / 2$ to all cells of the studies with zero 
非小细胞肺癌特异性。

\section{Summary Specificity}

Study | Spe [95\% Conf. Iterval. $] \quad T P /(T P+F N) \quad T N /(T N+F P)$

$\begin{array}{rllll}10.978 & 0.944 & -0.994 & 30 / 36 & 176 / 180 \\ 10.917 & 0.730 & -0.990 & 18 / 19 & 22 / 24\end{array}$

\begin{tabular}{l|lll} 
Pooled Spe & 0.971 & 0.937 & -0.989 \\
\hline
\end{tabular}

Pooled Spe $\quad 0.9710 .937-0.989$

$-----$

Heterogeneity chi-squared $=2.01($ d.f. $=1) \mathrm{p}=0.157$

Inconsistency $(I-$ square $)=50.2 \frac{0}{1+1}$

No. studies $=2$.

Filter OFF

Add $1 / 2$ to all cells of the studies with zero

非小细胞肺癌阳性似然比。

Summary Positive Likelihood Ratio (Random effects model)

Study | LRt [95\% Conf. Iterval.] $\%$ Weight

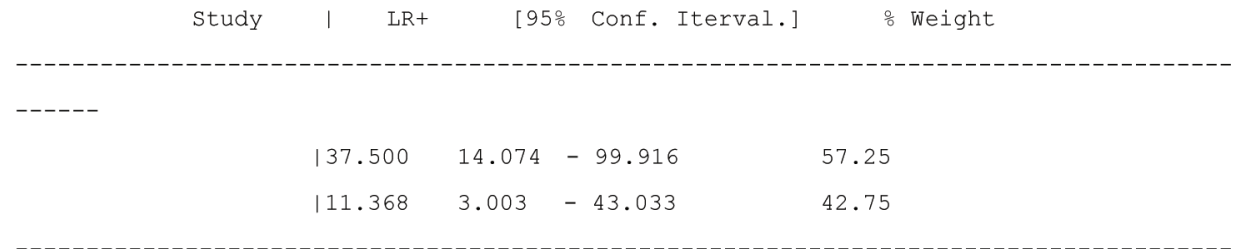

\begin{tabular}{l|lll} 
(REM) pooled LR+ & 22.513 & 6.983 & -72.578 \\
\hline
\end{tabular}

$-----$

Heterogeneity chi-squared $=2.05($ d.f.= 1) $p=0.152$

Inconsistency (I-square) $=51.2 \frac{0}{0}$

Estimate of between-study variance (Tau-squared) $=0.3731$

No. studies $=2$.

Filter OFF

Add $1 / 2$ to all cells of the studies with zero

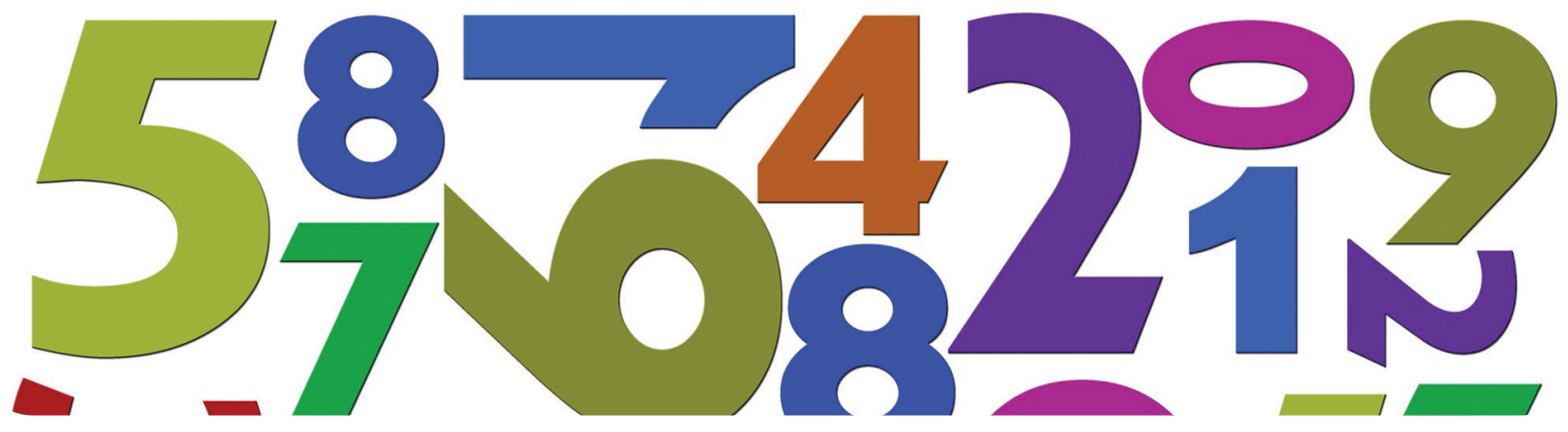


非小细胞肺癌阴性似然比。

Summary Negative Likelihood Ratio (Random effects model)

Study $\mid$ LR- [95\% Conf. Iterval.]

$\begin{array}{llll}10.170 & 0.082-0.354 & 83.85\end{array}$

$\begin{array}{llll}0.057 & 0.008-0.388 & -0.15\end{array}$

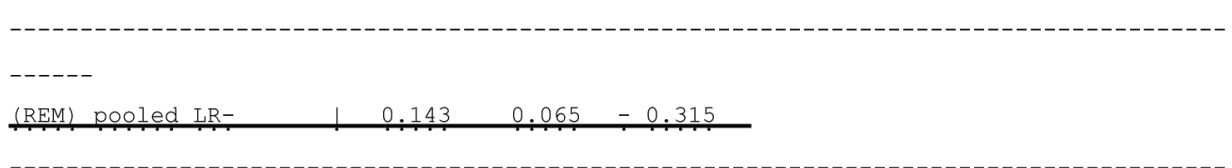

Heterogeneity chi-squared $=1.10($ d.f.= 1) $p=0.294$

Inconsistency (I-square) $=9.1 \frac{0}{9}$

Estimate of between-study variance (Tau-squared) $=0.0547$

No. studies $=2$.

Filter OFF

Add $1 / 2$ to all cells of the studies with zero

同样的我们可以得到:

小细胞肺癌敏感性

$>$ 小细胞肺癌特异性

$>$ 小细胞肺癌阳性似然比

$>$ 小细胞肺癌阴性似然比

现在我们在之前的最基本 的诊断性 meta 分析数据的基础 上可以得出非小细胞肺癌敏感性 (SEN)、特异性 (SPE)、阳性似
然比（PLR）、阴性似然比（NLR） 数据以及小细胞肺癌敏感性 (SEN)、特异性 (SPE)、阳性似 然比（PLR）、阴性似然比（NLR） 数据啦!

\section{结果是不是丰富一些了呢?}

关于基本的诊断学 meta 怎么 做的详细步骤, 可以翻炒写好诊 断性 meta 分析的秘密 (二 $)^{[1]}$ 。
关于更多的变化, 且听下回 分解。

参考文献

[1] 徐莉. 写好诊断性 meta分析的 的秘密(二). Medical Research \& Publication 2017;3(3):120-121. DOI:10.14218/MRP.2017.053.

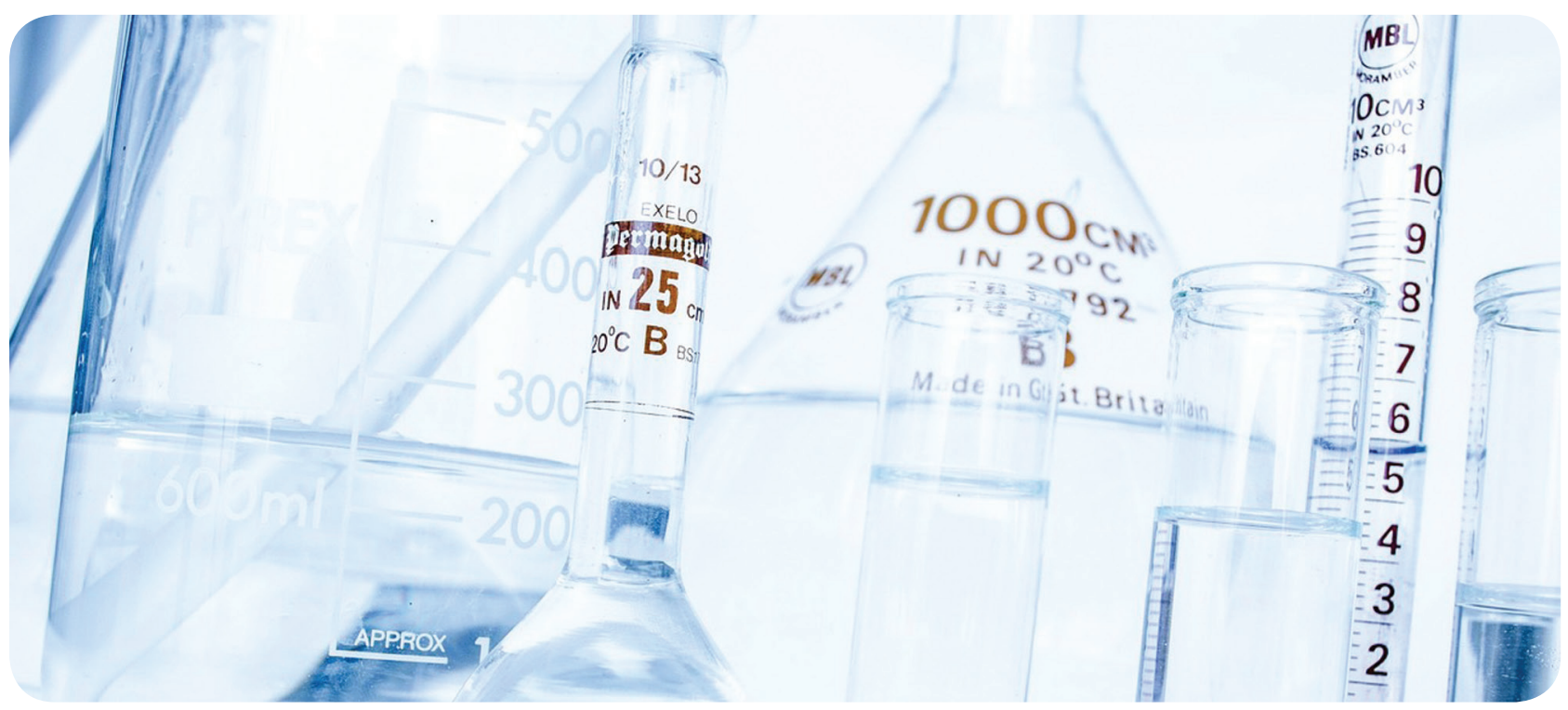

